\title{
Correction to: Two-Dimensional Culture Systems to Enable Mechanics-Based Assays for Stem Cell-Derived Cardiomyocytes
}

\author{
J. Notbohm ${ }^{1,2} \cdot$ B.N. Napiwocki ${ }^{2,3} \cdot$ W.J. de Lange ${ }^{4}$ A. Stempien ${ }^{2,3} \cdot$ A. Saraswathibhatla ${ }^{1} \cdot$ R.J. Craven ${ }^{2,3} \cdot$ \\ M.R. Salick ${ }^{3,5}$ - J.C. Ralphe ${ }^{4}$ - W.C. Crone ${ }^{1,2,3,5}$
}

Published online: 3 January 2020

(C) Society for Experimental Mechanics 2020

\section{Correction to: Experimental Mechanics(2019) 59:1235-1248} https://doi.org/10.1007/s11340-019-00473-8

The authors would like to correct the sentence "The mass ratio....." in the subsection on Engineered Substrate System in MATERIALS AND METHODS, to read:

The mass ratio of 1:50 produced a substrate with Young's modulus of approximately $10 \mathrm{kPa}$, while 1:10 produced $50 \mathrm{kPa}[60]$.

The online version of the original article can be found at https://doi.org/ 10.1007/s11340-019-00473-8

W. C. Crone

crone@engr.wisc.edu

J. Notbohm

jknotbohm@wisc.edu

1 Department of Engineering Physics, University of

Wisconsin-Madison, Madison, WI, USA

2 Department of Biomedical Engineering, University of Wisconsin-Madison, Madison, WI, USA

3 Wisconsin Institute for Discovery, University of Wisconsin-Madison, Madison, WI, USA

4 Department of Pediatrics, University of Wisconsin School of Medicine and Public Health, Madison, WI, USA

5 Department of Materials Science and Engineering, University of Wisconsin-Madison, Madison, WI, USA 